\section{P124 EFFUSION SIZE ON THE CHEST RADIOGRAPH AT DAY 7 POST-PLEURAL DRAINAGE IS A GOOD PREDICTOR OF SIZE AT 3 MONTHS, REGARDLESS OF INITIAL INTRAPLEURAL THERAPY}

doi:10.1136/thoraxjnl-2012-202678.407

'RJ Hallifax, 'M Nagendran, 'M Maruthappu, 'A Manuel, 'JM Wrightson, 'NA Maskell, ${ }^{2} \mathrm{HE}$ Davies, 'NM Rahman. 'Oxford Centre for Respiratory Medicine, Oxford, England; ${ }^{2}$ Department of Respiratory Medicine, Cardiff, Wales

Introduction Drainage of infection pleural fluid is essential in improving patient outcomes. The MIST-2 Trial (NEJM 2011) demonstrated intrapleural tissue plasminogen activator (t-PA) and DNase therapy improves drainage in patients with pleural infection from Day 1 to 7 compared to placebo. However, there is no evidence assessing whether this difference is sustained at 3 months. Methodology used in the MIST-2 trial demonstrated that a digital chest radiograph measurement strategy was an effective measurement of pleural fluid volume and correlated closely with computed tomography scanning in estimating effusion size.

Aims

1. To determine whether intrapleural treatment with t-PA, DNase, or both, showed a sustained significant difference in effusion size at 3 months.

2. To assess predictors of residual pleural shadowing 3 months post treatment.

Methods 132/193 (68\%) patients in the MIST-2 trial had assessable chest radiographs at 3 months (remaining patients were lost to follow-up or died). Effusion size was measured as a percentage of the hemithorax occupied on digital radiograph image (JPEG format). Each image was analysed independently by a medical student and respiratory specialist. Where the difference in estimates exceeded $2 \%$, the radiographs were reassessed together to generate consensus. Analysis was blind to treatment arm.

Results There was no significant difference between placebo and any treatment group in the 3 month radiology outcome. The 3 month effusion size was positively correlated with that at Day 7 (Pearsons $0.27, p=0.003$ ). Neither Day 1 effusion size, nor the difference between Day 1 to 7 was significantly correlated with 3 month outcome. Linear regression analysis demonstrated significant correlation for Day 7 effusion size with 3 months size $(p=0.004)$, but non-significance by treatment arm $(p=0.071)$.

Conclusions In those that survive and have a chest radiograph at 3 months, there is no difference in the radiological outcome at 3 months between treatment groups on the basis of treatments given, including surgical intervention. However, the appearance at day 7 is significantly correlated with the 3 month chest radiograph. Further statistical modelling of this data is now required to assess the relative contributions of intrapleural interventions and surgery in those that survive to 3 months.

\section{P125 DIGITAL MEASUREMENT STRATEGY IS ROBUST IN NON- EXPERT HANDS IN MEASURING CLINICALLY RELEVANT EFFUSIONS ON CHEST RADIOGRAPH}

doi:10.1136/thoraxjnl-2012-202678.408

M Nagendran, RJ Hallifax, M Maruthappu, A Manuel, NM Rahman. Oxford Centre for Respiratory Medicine, Oxford, UK

Introduction The primary outcome of most studies investigating pleural infection treatment is the pleural effusion volume change between two defined time points. This may be performed using thoracic computed tomography (CT) scans, but this method is costly and involves increased radiation to the patient. Data from the MIST-2 trial demonstrates that a digital chest radiograph measurement strategy was effective and correlated well with CT scanning.
Aim To assess deviation of novice scorers from an expert scorer when measuring pleural effusion size on chest radiographs using the digital scoring system.

Methods 132 chest radiograph digital images (JPEG format) were obtained from the 3 month follow-up of the MIST-2 trial. The optimal method was observer-estimated position of diaphragm and mediastinum with mediastinum discounted from the measurement. For each image, the area occupied by the pleural collection and hemithorax was manually drawn as a polyhedron, permitting calculation of percentage area occupied by effusion. Medical students acted as novice scorers and a respiratory specialist was the expert scorer and both independently scored the images.

Results Clinically significant effusions were defined as those over $2 \%$ of the hemithorax area. 73 of 132 were rated by the expert as not clinically significant. Of the remaining 59 , there was $>2 \%$ difference between novice and expert scores in 38 cases. Novice and expert then reassessed these radiographs together to agree a consensus. Within the subgroup of 59 clinically significant effusions, the mean difference between the novice and expert scores was $-0.10(95 \%$ CI -3.2 to $3.0, p=0.95)$. Comparison of novice and expert respectively to the final consensus scores revealed a significant mean difference in the novice scores of 2.0 (95\% CI 0.02 to 4.0, p $=0.048$, expert: $-0.11,95 \%$ CI -1.85 to $1.63, \mathrm{p}=0.90$ ).

Conclusions Novice scorers have a tendency to overestimate effusion size. However, where there is a clinically significant ( $>2 \%$ of hemithorax) effusion, scores between novice and expert correlate well. This implies that the digital measurement strategy is robust in non-expert hands in measuring clinically relevant effusions. This digital chest radiograph assessment tool is therefore a potential simple outcome for use in future studies assessing pleural drainage.

\section{P126 MEDICAL THORACOSCOPY EXPERIENCE IN A DISTRICT GENERAL HOSPITAL}

doi:10.1136/thoraxjnl-2012-202678.409

S Selvaraj, U Maqsood, M Chaudri, P Brammer. Russells Hall Hospital, Dudley, United Kingdom

Background In patients with pleural effusion, even after extensive diagnostic evaluation, the aetiology often remains unclear. The diagnostic yield from simple pleural fluid aspiration and blind pleural biopsy is very limited. Medical thoracoscopy can be performed under controlled sedation and enables direct visualisation of the pleural surfaces to obtain targeted sampling, to improve the diagnostic yield. ${ }^{1}$

Aim To review local practise and experience of medical thoracoscopy. Methods Retrospective analysis of 112 cases over a period of four years was performed to look at the diagnostic yield. We compared simple pleural aspiration with thoracoscopy and examined the relationship between the computed tomography (CT) appearances and histology results.

Results Of the 112 cases, 83 (74\%) were male and 27 (26\%) female. The age range was 32 to 90 years (mean - 69). No major adverse events were reported following the procedure. Two patients had the procedure performed twice due to recurrence. One sample was lost. The results for the remaining 111 patients are presented in the table.

Of the 69 patients who were diagnosed with malignancy, pleural fluid aspiration was positive in only $29(43 \%)$ cases. CT evidence of pleural thickening was noted in only 28 (41\%) of these cases, all of which turned to be malignant.

\section{Conclusions}

1. Our study reiterates the fact that medical thoracoscopy has a better diagnostic yield than simple pleural aspiration and is a safe procedure that can be performed safely by chest physicians in a DGH setting. 
2. In our case series the presence of pleural thickening on CT was a strong predictor of malignancy. However the majority of malignant cases had no evidence of pleural thickening.

\section{Reference.}

1. BTS pleural disease guideline -2010 .

Abstract P126 Table 1

\begin{tabular}{ll}
\hline Diagnosis & Numbers (\%) \\
\hline MALIGNANT & $69(62 \%)$ \\
Adenocarcinoma & 21 \\
Mesothelioma & 19 \\
Metastasis & 13 \\
Lymphoma & 7 \\
Squamous cell carcinoma & 4 \\
Sarcoma & 2 \\
Neuro endocrine tumours & 3 \\
BENIGN PLEURAL INFLAMMATION & $40(36 \%)$ \\
SARCOIDOSIS & $1(1 \%)$ \\
TUBERCULOSIS & $1(1 \%)$ \\
\hline
\end{tabular}

\section{Interstitial lung disease: epidemiology, care and survival}

\section{P127 PREVALENCE AND INCIDENCE OF IDIOPATHIC PULMONARY FIBROSIS IN UK HEALTHCARE DATABASES, GPRD AND THIN; THE NEED FOR AN IPF REGISTRY}

doi:10.1136/thoraxjnl-2012-202678.410

'PL Thompson, ${ }^{2} \mathrm{~S}$ Chretin, ${ }^{2} \mathrm{~F}$ Bugnard, ${ }^{1} \mathrm{WC}$ Maier, ${ }^{3} \mathrm{R}$ Cameron, ${ }^{3} \mathrm{M}$ Fisher, ${ }^{3} \mathrm{C}$ Giot, ${ }^{3} \mathrm{C}$ Hill, ${ }^{3}$ Kausar. 1Registrat-Mapi, Hamilton House - Office 322, Mabledon Place, Bloomsbury, WC1H 9BB, London, UK; ${ }^{2}$ Registrat-Mapi, 27 rue de la Villette, 69003 Lyon, France; ' IInterMune UKIreland, Euston Tower - Floor 32, 286 Euston Road, NW1 3DP, London, UK

Background Idiopathic Pulmonary Fibrosis (IPF) is a fatal respiratory illness with limited treatment options.

We previously estimated the UK prevalence of IPF using The Health Improvement Network (THIN) data. We have now further investigated this using General Practise Research Database (GPRD) data.

Aims Calculate the prevalence and incidence of IPF diagnoses in the GPRD in 2010.

Calculate the prevalence of IPF diagnoses in the GPRD in 2007, to compare to the THIN 2007 findings.
Build a matrix of epidemiological data, to better target future public health resources.

Methods Descriptive cross-sectional study, using UK GPRD data to calculate the prevalence and incidence of IPF diagnoses in 2010.

Patients with a Read/OXMIS code corresponding to IPF diagnosis were identified; H563.00 Idiopathic Fibrosing Alveolitis (IFA), H563z00 Idiopathic Fibrosing Alveolitis NOS (IFA NOS), H563.12 Cryptogenic Fibrosing Alveolitis (CFA), H563100 Diffuse Pulmonary Fibrosis (DPF). To investigate coding variability, IPF diagnoses were classified as broad (all codes), narrow (IFA/IFA NOS) and IFA+CFA

Patients with a first record of IPF during 2010 were classed as incident. Patients with a first record of IPF prior to or during 2010 were classed as prevalent.

Results In 2010 in the GPRD, IPF was most commonly diagnosed in males (56.5\%) and $\geq 65$ year olds (80.8\%). Most IPF was coded as DPF (prevalence: 39.4 [37.6-41.3] per 100,000 persons; incidence: 9.6 [8.8-10.6] per 100,000 person-years).

For the broad definition, IPF prevalence was 50.7 (48.6-52.8) per 100,000 persons, with an incidence of $11.0(10.1-12.0)$ per 100,000 person-years. For the narrow definition, IPF prevalence was 10.1 (9.2-11.1) per 100,000 persons, with an incidence of 1.5 (1.1-1.9) per 100,000 person-years. For the IFA+CFA definition, IPF prevalence was 13.3 (12.3-14.4) per 100,000 persons, with an incidence of $1.6(1.3-2.0)$ per 100,000 person-years.

In 2007, compared to THIN, the GPRD IPF point prevalence was lower in both males and females, for all diagnosis definitions (Figure 1).

Conclusions IPF rates differ amongst UK healthcare databases; this may be due to GP coding variability, which is an inherent limitation of database studies. A prospective IPF registry is essential to characterise this orphan disease, to better target future public health resources.

\section{P128 PRACTISE PATTERNS IN IDIOPATHIC PULMONARY FIBROSIS: RESULTS OF A UK PHYSICIAN SURVEY}

doi:10.1136/thoraxjnl-2012-202678.411

'I Kausar, 'R Cameron, NC Eastmond. 'InterMune UK\&l, London, United Kingdom; 'Eastmond Medicomm Ltd, High Peak, United Kingdom

Introduction and objectives Idiopathic pulmonary fibrosis (IPF) is a rare, progressive, fibrotic lung disorder that results in reduced lung capacity, disability and ultimately death. Its rarity, and the fact that many of its initial symptoms are common to other lung diseases, mean that it is often mis-diagnosed and patients can experience wide variations in standards of care. We conducted a survey of

Figure 1: Gender- and diagnosi s-specific point prevalence of IPF in 2007, GPRD compared to THIN

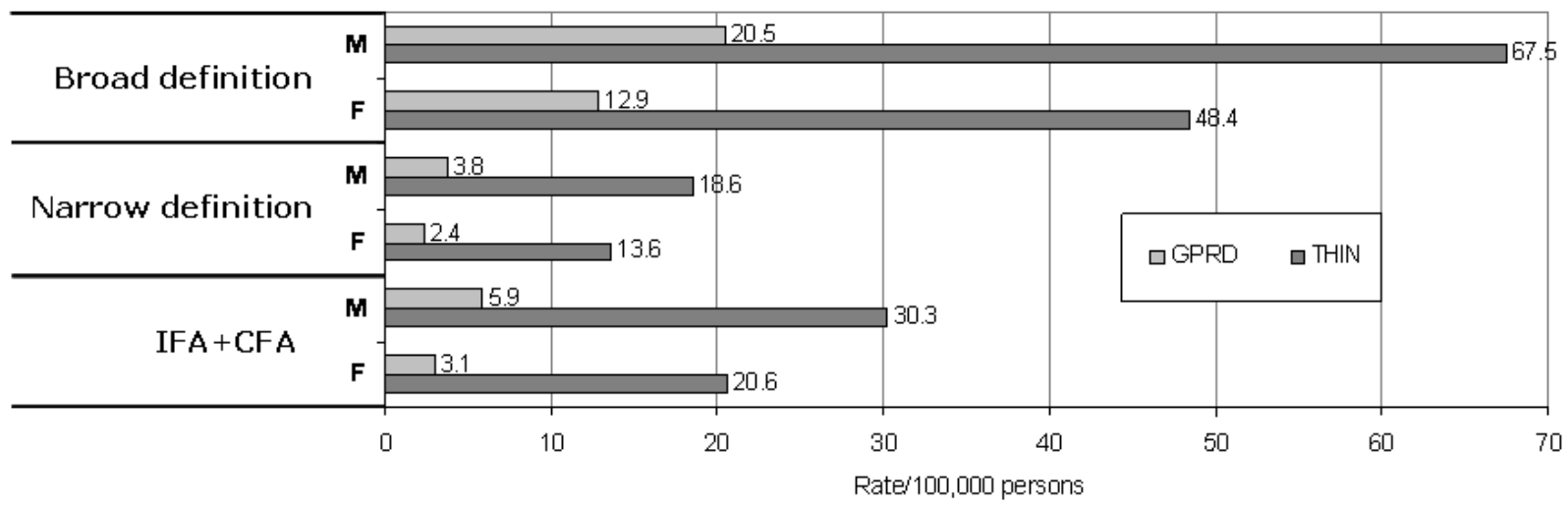

